\title{
Time scale analysis for fluidized bed melt granulation- II: binder spreading rate
}

Kel W. Chua ${ }^{a}$, Yassir T. Makkawi ${ }^{b, *}$, Buddhi N. Hewakandamby ${ }^{c}$, Michael J. Hounslow ${ }^{a}$

a Particle Products Group, Department of Chemical and Process Engineering, The University of Sheffield, Sheffield S1 3JD, United Kingdom

b Chemical Engineering and Applied Chemistry, School of Engineering and Applied Science, Aston University, Birmingham B4 7ET, United Kingdom

c Department of Chemical and Environmental Engineering, Faculty of Engineering, University of Nottingham, Nottingham NG7 2RD, United Kingdom

\section{Abstract}

The spreading time of liquid binder droplet on the surface a primary particle is analysed for Fluidized Bed Melt Granulation (FBMG). As discussed in the first paper of this series (Chua et al., 2010a) the droplet spreading rate has been identified as one of the important parameters affecting the probability of particles aggregation in FBMG. In this paper, the binder droplet spreading time has been estimated using Computational Fluid Dynamic modeling (CFD) based on Volume of Fluid approach (VOF). A simplified analytical solution has been developed and tested to explore its validity for predicting the spreading time. For the purpose of models validation, the droplet spreading evolution was recorded using a high speed video camera. Based on the validated model, a generalized correlative equation for binder spreading time is proposed. For the operating conditions considered here, the spreading time for Polyethylene Glycol (PEG1500) binder was found to fall within the range of $10^{-2}$ to $10^{-5} \mathrm{~s}$. The study also included a number of other common binders used in FBMG. The results obtained here will be further used in paper III, where the binder solidification rate is discussed.

Keyword: fluidization; granulation; binder spreading, VOF modeling; particle processing; particle technology

\footnotetext{
* Corresponding author. +44 (0)121 204 3398; email: y.makkawi@aston.ac.uk
} 


\section{Introduction}

In Fluidized Bed Melt Granulation (FBMG) processes, primary particles are fluidized with air while hot molten liquid binder is sprayed into the fluidized bed. The binder droplets come into contact with the primary particles or granules and rapidly spread before solidifying on the particle surface. If a particle or granule collides with another, while some of the binder is wet, the two might stick, thus giving rise to an increase in size.

The granulation rate is related to the granule-granule collision rate, droplet spreading rate and droplet solidification rate. In series I of this study we have shown that granule-granule collision rate in a typical FBMG occurs in a time scale in the range of 0.01 second. In this series, we are mainly interested in identifying the liquid droplet spreading time and the corresponding final spreaded area at an equilibrium state. If the binder spreading rate is much faster than the granule-granule collision rate, the droplet will have sufficient time to spread over the particle surface before collision occurs, hence, the aggregation probability is higher. On the other hand, if the binder spreading rate is much slower than granulegranule collision rate, the primary particles are more likely to collide on a dry surface, hence, reducing the aggregation efficiency.

Binder spreading time relative to binder solidification time determines the dynamic of binder wetting process. If the spreading time scale is shorter than solidification time scale, the binder droplet will be able to spread to its equilibrium contact angle. If spreading time scale is longer than solidification time scale, the binder droplet will start to solidify before the droplet completes the spreading process. In the second case, the application of equilibrium contact angle to predict wetted area per droplet will not be appropriate. Therefore, it is necessary to determine spreading time scale in order to gain better understanding of the granulation process.

Spreading of macroscopic drops of Newtonian liquids on smooth surfaces has been studied experimentally and theoretically in detail during the past few decades (Tanner, 1979; Chen, 1988; Brenner and Bertozzi, 1993; Rafaï et al. 2004). Tanner (Tanner 1979) described the evolution of the droplet radius, $d$ on a planar surfaces as function of time, 
$d=C t^{n}$, where the value of the power $n$ depends on the dominance of forces on spreading. When capillarity dictates the spreading $n=1 / 10$, while the gravity dominates when $n=1 / 8$. Experimental evidence (Ausserré, 1986; Cazabat and Cohen-Stuart, 1986; Chen, 1988) supports the universality of Tanner's laws. Rafaï et al. (2004) showed that Tanner's law could also be derived from droplet spreading measurements of nonNewtonian polymer droplets.

Modeling of liquid droplet spreading on solid surfaces has been studied in the past, particularly that of relevance to the field of printer inkjet printing (e.g. Clarke et al., 2001). This was usually modeled in two dimensions using lubrication approximations where the flow is heavily simplified (Hocking, 1992). Nowadays, there are many efforts to model the spreading characteristics computationally using fully nonlinear system equations such as the Volume of Fluid (VOF) method (e.g. Gopala and van Wachem, 2008) and the Overall Energy Balance (OEB) method (e.g. Gu and Li 1998). The OEB method models liquid spreading process on solid surfaces by considering the potential energy due to interfacial tension and kinetic energy balanced by the energy dissipation during spreading. The VOF, on the other hand, includes four common methods: (1) Fluxcorrected transport by Boris and Book (1973), (2) Lagrangian piecewise linear interface construction by van Wachem and Schouten (2004), (3) Compressive interface capturing scheme for arbitrary meshes (CICSAM) by Ubbink(1997) and (4) inter-gamma scheme by Jasak and Weller (1995). Each of these methods employs a different approach to track the phase interface. In a recent comprehensive review by Gopala and van Wachem (2008) it was shown that the CICSAM and inter-gamma scheme are the most accurate in conserving mass and tracking the interface. CICSAM and inter-gamma scheme were also found to be flexible in terms of arbitrary mesh usage, as they do not require direction splitting for higher geometric dimensions.

In this paper, liquid binder droplet spreading is studied using three different complementary methods; a commercial CFD package (Fluent Ver. 6.3), high speed digital video recording and "theoretical" dimensional analysis. The binder spreading on flat surface is simulated using CICSAM VOF method built in the Fluent package. The 
CFD predictions are compared with the spreading behavior of Polyethylene Glycol (PEG1500) droplet captured by a high speed camera. The validated model is then used to simulate the droplet spreading behavior on a smooth curved surface, in a similar scale and condition typically found in a spray FBMG. Finally, a dimensional analysis is conducted to derive a predictive equation of the spreading rate in terms of the binder physical parameters and the primary particle size. Using this equation, the binder spreading time for different binder characteristics in FBMG processes is presented.

\section{Simplified theory}

We wish to know the time required for a drop of binder, of diameter $d_{o}$, to spread over the surface of a single primary particle, of diameter $D_{p}$ (see Fig 1). For this purpose, we propose that the spreading process is driven by the effective surface tension, $\gamma \cos \theta$, and resisted by the viscosity of the binder, $\mu$. In Fig. 1, we represent the spreading drop as a cylinder of radius, $a$, and height, $h$, and neglect the curvature of the granule. The surface tension force acting to spread the $\operatorname{drop}$ is $2 \pi a \gamma \cos \vartheta$. The velocity gradient of the liquid in the drop is approximately $\dot{a} / h$, so the shear stress is $\mu \dot{a} / h$, and the retarding force $\pi a^{2} \mu \dot{a} / h$. Neglecting inertia requires that these two forces be equal such that $2 a \gamma \cos \vartheta=a^{2} \mu \dot{a} / h$, which can be combined with a statement of conservation of volume $a^{2} h=d_{0}^{3} / 6$, to give,

$$
\frac{d a}{d t}=\frac{d_{0}^{3} \gamma \cos \vartheta}{3 \mu a^{3}}
$$

Spreading ceases when the spreading front reaches equilibrium contact angle. Spreaded radius at equilibrium, $a_{f}$ can be approximated using the geometrical model of a droplet at equilibrium resting on a flat surface proposed by Clarke et al. (2001):

$\frac{d_{f}}{2}=\left[\frac{3 V_{d}}{\pi} \frac{\sin ^{3} \vartheta}{\left(2-3 \cos \vartheta+\cos ^{3} \vartheta\right)}\right]^{1 / 3}$ 
where $d_{f}$ is the final spreaded diameter, $V_{d}$ is the droplet volume and $\vartheta$ is liquid-solid contact angle. For a given liquid-solid contact angle, $d_{f}$, is proportional to $d_{o}$, thus, Eq. 2 reduces to

$d_{f}=K_{w} d_{o}$

where $K_{w}$ is the constant of proportionality given as function of the contact angle as follows:

$K_{w}=2\left[\frac{0.5 \sin ^{3} \vartheta}{\left(2-3 \cos \vartheta+\cos ^{3} \vartheta\right)}\right]^{1 / 3}$

Now, in integrating Eq. 1 , we take the initial condition to be $a_{0}=d_{0} / 2$, and the final condition to be $a_{f}=K_{w} d_{o} / 2$, this gives a spreading time,

$\tau_{w}=\frac{3 \mu}{64 \gamma \cos \vartheta} d_{o}\left(K_{w}^{4}-1\right)$

For PEG1500, at around $55^{\circ} \mathrm{C}$, we measured the contact angle to be $\vartheta=35^{\circ}$, giving $K_{w}=2$, thus according to Eq. 3, the diameter of the final wetted area is $d_{f}=2 d_{o}$. Substituting for a droplet size of $40 \mu \mathrm{m}$ diameter, along with the PEG1500 physical parameters given in Table 1, Eq. 5 gives a spreading time of $9.5 \times 10^{-6}$ seconds.

Since the above represents a simplified solution, which assumes constant physical properties of the spreading binder and neglects the effect of particle curvature and $\vartheta$, we seek to explore more accurate solution by numerical simulations as shown in the following section.

\section{Simulation}

\subsection{VOF model (Fluent)}

The so called "Volume of Fluid" model, built in the Fluent simulation package, was used to simulate the binder spreading process. The crux of the VOF method is to track the 
binder-air interface, without inter-penetration of the two media, by solving continuity, momentum and energy equations within a control volume with bulk phase of binder and air. The continuity equation for the dispersed phase volume fraction $\alpha_{l}$ within the control volume can be written as follows:

$\frac{1}{\rho_{l}}\left[\frac{\partial}{\partial t}\left(\alpha_{l} \rho_{l}\right)+\nabla \cdot\left(\alpha_{l} \rho_{l} \vec{v}_{l}\right)=0\right]$

In the problem considered here, Eq. 6 is solved for the both dispersed phases, i.e. the liquid binder and air, and the volume fraction of the continuous phase is computed such that the sum of all volume fractions in each cell is unity. This allows the estimation of physical properties within each cell. For instance, the effective density and viscosity are obtained as follow:

$\rho=\alpha_{l} \rho_{l}+\left(1-\alpha_{l}\right) \rho_{g}$

$\mu=\alpha_{l} \mu_{l}+\left(1-\alpha_{l}\right) \mu_{g}$

This approach permits a single momentum balance equation throughout the computational domain providing the coupling between the volume fractions and the flow through local physical properties. The momentum balance equation,

$\frac{\partial}{\partial t}(\rho \vec{v})+\nabla \cdot(\rho \vec{v} \vec{v})=-\nabla p+\nabla \cdot\left[\mu\left(\nabla \vec{v}+\nabla \vec{v}^{T}\right)\right]+\vec{F}$

is solved within the domain. The viscosity $\mu$ and density $\rho$ are effective values computed using Eq. 7. Note that in Eq. 8 the gravity force is omitted, because the droplet length scales are below the capillary length scale. The term $\vec{F}$ in Eq. 8 represent volume forces. Following Brackbill et al. (1992), the surface tension forces, modeled using Continuum Surface Force (CSF) method, is implemented in the momentum equation as a volume force via divergence theorem. This approach gives,

$$
\vec{F}=\gamma \frac{\rho \kappa \nabla \alpha_{l}}{0.5\left(\rho_{l}+\rho_{g}\right)}
$$

where $\kappa$ is the curvature of the interface. Since the interface is tracked through the propagation of the volume fraction $\alpha_{l}$, the curvature, $\kappa$ can be defined as, 
$\kappa=\frac{\nabla \alpha_{l}}{\left|\nabla \alpha_{l}\right|}=\nabla \cdot \frac{\hat{n}}{|\hat{n}|}$

where $\hat{n}$ is the surface normal vector.

Liquid volume fraction at the liquid-air interface is tracked using CICSAM-scheme (Compressive Interface Capturing Scheme for Arbitrary Meshes) proposed by Ubbink's (1997). Surface adhesion of the binder (liquid phase) forms a contact line with contact angle $\vartheta$. Following Brackbill et al. (1992), the contact angle is used to estimate the curvature of the surface near the wall. The surface normal at the live cell next to the wall is defined using the contact angle as follows:

$\hat{n}=\hat{n}_{w} \cos \vartheta+\hat{t}_{w} \sin \vartheta$

where $\hat{n}_{w}$ and $\hat{t}_{w}$ are the unit vectors normal and tangential to the wall, respectively. The surface normal vector is used to determine the local curvature of the interface used in Eq. 10.

A heat transfer model was incorporated in the VOF solution to track the temperature changes in the spreading liquid binder. One energy equation is shared among the phases, as shown below:

$\frac{\partial}{\partial t}(\rho E)+\nabla \cdot(\vec{v}(\rho E+P))=\nabla \cdot\left(k_{\text {eff }} \nabla T\right)$

where isothermal boundary condition is implemented on the granule surface. The effect of temperature variation on the binder physical properties (except on binder viscosity) are assumed negligible.

Eqs. 6 to 12 are all solved simultaneously using Fluent (version 6.3) simulation package. Similar application of Fluent, where liquid droplet spreading behavior is studied numerically, can be found in Gunjal et al. (2003) and Lunkad et al. (2007). This study however, is a rather more complicated case in which a droplet at a microscale level undergoes simultaneous dynamic spreading and solidification in a typical range of operating conditions considered in fluidized bed granulation. 


\subsection{Simulation procedure}

Spreading of a spherical liquid droplet of PEG1500 on a flat and curved surface is considered in this simulation. The simulations on the flat surface are used to validate the VOF model with experimental data. The validated model is then considered for curved surface to mimic the spreading of liquid droplet on the surface of a suspended particle in a typical droplet and particle sizes that exist in a FBMG.

The simulation of a droplet touching a planar glass surface was carried out using a three-dimensional rectangular box with a Cartesian grid. The grid size is set at 10 cells per radius. Previously Bussmann (2000) studied the effect of the grid resolution using 10, 16 and 25 cells per radius, where it was concluded that a grid resolution of 10 cells per radius was sufficient to capture accurately the dynamics of droplet spreading. The computational domain size used in the $x, y, z$ coordinates is $2 d_{o}, 2 d_{o}$, and $1.15 d_{o}$ respectively, where $d_{o}$ represents the initial droplet diameter.

In the model boundaries, no slip condition is specified at the spreading surface and all the remaining faces are defined as pressure inlets. Also on the spreading surface, isothermal boundary condition is specified and the liquid-surface static contact angle (determined from experimental measurement) was imposed in the model solution.

The viscosity of PEG1500 is incorporated in the model using experimentally determined correlation as discussed in section 4.1. Following Bicerano (2002), the surface tension of PEG1500 is considered to be independent of temperature.

\section{Validation experiment}

\subsection{Experimental set up and procedure}

PEG1500 (supplied by BP Chemicals, UK) is solid at room temperature; therefore the solid flakes were heated to the desired temperature above the melting temperature in a 
controlled reservoir using a hot plate. Two different initial droplet temperatures of $50^{\circ} \mathrm{C}$ and $67^{\circ} \mathrm{C}$ were considered. A glass plate, cleaned with acetone and water and dried with lint-free tissue, was used as the spreading surface. The droplet of the melt was dispensed using a calibrated syringe, $0.97 \mathrm{~mm}$ inside diameter (KDS1812P, Kahnetics) and allowed to fall freely from the height of $3 \mathrm{~cm}$ above the flat glass surface. A high speed camera (FastCam Pro1024, Photron) was orientated toward the glass surface to capture the evolution of the droplet spreading. A total of 2000 images were captured at a frame rate of 500 frames per second. The resolution of the camera used was $1024 \times 512$ pixels. For illumination, a soft light with color temperature scale of 3100K (KL1500-LCD, Zeiss) was placed facing the camera lens and directly behind the spreading area to enhance the image quality. The experimental arrangement is shown in Fig. 4.

The variation of PEG1500 viscosity as function of temperature was determined experimentally using magnetic bearing rheometer (AR-G2 Rheometer, TA Instruments, USA). For this purpose, $4 \mathrm{mg}$ of solid PEG1500 flakes was used. From experiments with $1 / 10$ to $1 / 1000$ shear rates, viscosity of the liquid PEG1500 was found to be independent of the shear rate. The variation of viscosity as function of temperature at an applied shear rate of $1 / 10 \mathrm{~s}^{-1}$ is shown in Fig. 5. This experimental data was fitted to a power law function to give the PEG1500 viscosity as function of temperature.

\subsection{Data analysis method}

In analyzing the liquid droplet spreaded area, a threshold method was used to determine the droplet diameter and height from the captured images. The images were loaded into image processing software (ImageJ, version 1.38x) to segment each image into droplet area and background. This was done by identifying the pixels with grayscale values greater than the droplet region. The total identified segments compromise a spherical cap shape which was then used to determine the instantaneous diameter of the spreading droplet in a given snapshot. 


\subsection{Validation of the VOF model}

In order to mimic the experimental conditions, the numerical simulations were considered for the exact initial droplet size and initial temperature used in the experiment. The simulation results for a droplet spreading on a flat glass surface in comparison with experimentally captured images are shown in Fig. 6. It is worth noting that in the experiments, the droplet was left to fall freely to the surface from a very close distance, such that the inertia of impact can be safely ignored. The results shown in Fig. 6 show a remarkable qualitative agreement between the experimental images and the simulation.

A quantitative validation of the model predictions is shown in Fig. 7. The variation of the spreaded distance, expressed in terms of $d / d_{o}$, is shown as function of time for two different initial droplet temperatures. $d / d_{o}$ is the ratio of dynamic to initial diameter of the projected droplet cross-section. In these figures, the final spreaded area is higher for higher initial droplet temperature. $d / d_{o}$ is found to fall between 1.9 and 1.8 for the initial temperature range of $50^{\circ}$ and $67^{\circ}$ respectively. Despite the slight discrepancy between the model and experiment at early stage of spreading shown in Fig. 7b, the model provides reasonably accurate description of the spreading behavior and hence accurate estimation of the spreading time given that the melt temperature is high enough to reach the equilibrium contact angle before solidification.

Here, there is an argument that, the spreading progression might have ceased due to binder solidification. However, the predicted temperature profile at the liquid bindergranule surface interface shown in Fig. 8 indicates less than $20^{\circ} \mathrm{C}$ drop in temperature throughout the spreading time, thus eliminating any possibility of solidification within this time scale. In part III of this series, our experimental evidence, and indeed the simulation results for solidification time of PEG1500 droplet, confirm a rapid spreading with the time scale well below the solidification time scale.

It is also interesting to note that in Fig. 7, the spreading behavior can well be described by the simple exponent law of Tanner, $d=C t^{n}$, where the exponent $n$ and coefficient $C$ 
are functions of the initial droplet volume or mass (Tanner, 1979; Summ and Samsonov, 1999). From these figures, it can be deduced that the exponent coefficient $n$ falls within the range of $0.9-0.13$, which is reasonably close to the values reported in Summ and Samsonov (1999). The coefficient $C$ can be expressed in terms of the droplet mass, such that $C=M^{p}$, where the exponent $p$ deduced from these figure is found to be equal to 0.53 .

\section{Simulation results}

\subsection{Effect of contact angle}

Fig. 9 shows the simulation results of the time required to reach equilibrium versus $\cos \theta$ for various contact angles ranging from $30^{\circ}$ (high spreading) to $120^{\circ}$ (non-spreading). A straight line appears to provide a good fit, indicating that the spreading time is proportional to the power law $(\cos \theta)^{1.53}$. The spreading time for a small contact angle is longer because the binder has a larger spreading distance before the liquid front reaches equilibrium. For large contact angle (non-spreading), the time required to reach equilibrium is extremely fast due to the limited spreading distance.

\subsection{Effect of particle curvature $\left(D_{p} / d_{0}\right)$}

In FBMG, the initial droplet size can be varied via the atomizing air pressure. Therefore a range of droplet size usually considered in FBMG process has been used here. Fig. 10 shows simulation results of the spreading time versus a range of particle diameter to initial droplet size ratios, $D_{p} / d_{o}$. Here, the spreading time appear to linearly decrease with increasing the ratio $D_{p} / d_{o}$. This could be attributed to (i) curvature effect at decreasing particle size (ii) inertia effects at increasing droplet size. A straight line of slope equal to -0.5 appear to fit well the simulation data, indicating that the spreading time is inversely proportional to the square root of $D_{p} / d_{o}$. 


\subsection{Effect of binder surface tension}

The surface tension has a direct effect on the spreading rate, since the liquid front velocity is proportional to $\gamma \cos (\vartheta)$. Therefore, a higher surface tension implies a faster spreading rate. Fig. 11 shows the simulation results of spreading time as function of $1 / \gamma$ for various binder surface tension values between 0.01 and $1.0 \mathrm{~N} / \mathrm{m}$. From these simulation data, a straight line was fitted, indicating that the spreading time is linearly dependent on the inverse of the surface tension.

\subsection{Effect of binder viscosity}

Opposite to the effect of surface tension, binder viscosity is a retarding force working against the liquid spreading. Fig.12 shows the simulation results of spreading time for a range of binder viscosities between 0.01 and 1.0 Pa.s. From the data, the spreading line appears to be directly proportional to the viscosity.

\section{Generalized correlation of spreading time}

The simplified theory presented in section 2 suggests that the spreading time, $\tau_{w}$ may be related to the following variables:

$\tau_{w}=f\left(\vartheta, d_{o}, D_{p}, \mu, \gamma\right)$

Using dimensional analysis and the results of numerical simulation shown in section 5, we propose that, $\tau_{w}$ can be expressed in terms of dimensionless variables, such that:

$\tau_{w}=f\left[(\cos \vartheta)^{1.53},\left(\frac{D_{p}}{d_{0}}\right)^{-0.5},\left(\frac{d_{o} \mu}{\gamma}\right)\right]$

or

$\tau_{w}=K_{W}\left[(\cos \vartheta)^{1.53}\left(\frac{D_{p}}{d_{0}}\right)^{-0.5}\left(\frac{d_{0} \mu}{\gamma}\right)\right]$

where $K_{W}$ is a constant to be found by plotting the simulation results for the spreading time against the value of the term between the main bracket in Eq. 15. For the whole 
range of conditions considered in Table 2, Fig. 13, shows that the constant of proportionality is $K_{W} \approx 29.0$.

Figs. 14a-d show the spreading time calculated from the above generalized correlation in comparison to the values obtained from the VOF model and the simplified analytical solution given by Eq. 5. The proposed generalized correlation (Eq. 15) proved to provide a good match with the spreading time predicted by the VOF model over a wide range of operating conditions. The analytical solution, however, underestimates the spreading time, roughly by a factor of 10 . This deviation appears to slightly increase as the droplet size increases as shown in Fig. 14a. This may be attributed to the fact that the proposed analytical solution ignores the effects of the particle curvature and droplet inertia, which both becomes important as the droplet size becomes large relative to the particle size. Another factor which may have contributed to this deviation is that in the simplified analytical solution, the viscosity, as well as the surface tension, is assumed constant, while the VOF model incorporates accurate viscosities in term of temperature variations. A change in viscosity, as a result of heat loss, leads to slower binder front velocity; consequently, the analytical model underestimates the spreading time compared to the VOF predictions.

\subsection{Spreading time for commonly used binders in FBMG}

PEG1500, CMC and HPC are common binders used in granulation of tablets. Using the generalized correlation of Eq. 15, Table 3 shows the spreading time for a droplet size of $40 \mu \mathrm{m}$ spreading over a $200 \mu \mathrm{m}$ diameter particle. The values of surface tension, contact angle and viscosity were obtained from reported literature. The spreading time for all binders considered is found to fall within the range of $10^{-5}$ to $10^{-3}$ seconds.

Recalling the particle-particle collision rates discussed in first part I of this study $\left(2.5 \times 10^{-3}\right.$ to $10^{-2} \mathrm{~s}$ ), the spreading rates for all of the above discussed binders are sufficient to ensure rapid binder spreading rate in a fluidized bed granulator. However, any deviations in the binder physical properties from the ranges considered here, such as increasing the viscosity beyond 0.2 Pa.s or decreasing the surface tension below 0.01 
$\mathrm{N} . \mathrm{m}^{-1}$ may result is significantly longer spreading time, thus, limiting the overall granulation efficiency. For successful aggregation, it is desirable to have rapid spreading relative to collision and solidification rates.

\section{Conclusions}

In this paper, the binder spreading time in the context of fluidized bed granulation process is analyzed using an experimentally verified computational fluid dynamic modeling based on Volume of Fluid approach (VOF). A simple analytical solution is also developed. The VOF predictions are validated with experimental data obtained from high speed video recording of droplet spreading.

A generalized relationship for binder spreading time as function of contact angle, droplet size, binder contact angle, surface tension and viscosity is obtained by conducting a series of parametric analysis using the VOF predictions. Based on this generalized relationship, the spreading time for a number of commonly used binders in FBMG (PEG1500, CMC 1\% and HPC 5-10\%) is estimated to fall within the range of $10^{-5}$ to $10^{-3}$ s. Due to its simplicity, and the fact that it neglects the particle curvature and possible variation in spreading binder physical properties, the proposed analytical solution under estimates this spreading time, roughly by a factor of 10 . However, this robust method is a viable tool for establishing relation between spreading time and operating conditions for a microscopic phenomenon that may well be extremely difficult to measure experimentally. 


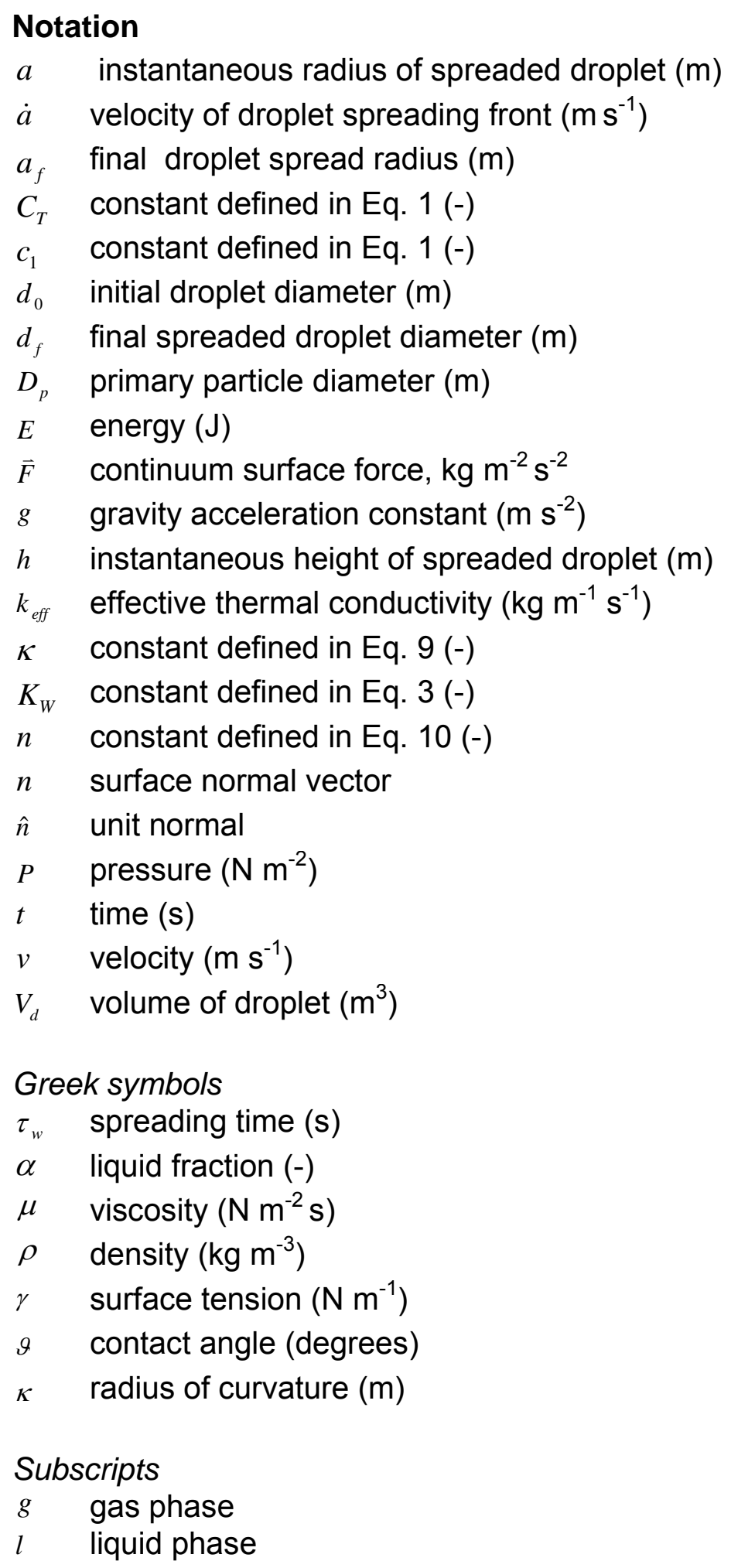




\section{References}

Ausserré, D., Picard, A. M., and Léger, L., 1986. Existence and Role of the Precursor Film in the Spreading of Polymer Liquids._Phys. Rev. Lett, 57, 2671-2674.

Bicerano, J., 2002. Prediction of polymer properties. Midland, Michigan, USA, CRC Press.

Boris, J. P. and Book, D. L., 1973. Flux-corrected transport. I. SHASTA, a fluid transport algorithm that works. Journal of Computational Physics, 11, 38-69.

Brackbill, J.U., Kothe, D.B., Zemach, C., 1992. A continuum method for modeling surface tension. Journal of Computational Physics, 100, 335-354.

Brenner, M. and Bertozzi, A., 1993. Spreading of Droplets on a Solid Surface. Phys. Rev. Lett., 71, 593-96.

Bussmann, M., 2000). A three-dimensional model of an impacting droplet. Ph.D. Thesis. Department of Mechanical and Industrial Engineering, University of Toronto, Canada.

Cazabat, A. M. and Cohen-Stuart, M. A. 1986. Dynamics of wetting: effects of surface roughness. J. Phys. Chem., 90, 5845-5849.

Chen, D. J., 1988. Experiments on a spreading drop and its contact angle on a solid. J. Colloid and Interface Science, 122, 60-72.

Clarke, A., Blake, T. D., Carruthers, K., Woodward, A., 2001. Spreading and Imbibition of Liquid Droplets on Porous Surfaces, Langmuir, 18, 2980 -2984.

Chua, Kel W., Makkawi, Y. T., Hounslow, M. J., 2010a. Time scale analysis for fluidized bed melt granulation I: granule-granule and granule-droplet collision rates. Chemical Engineering Science, submitted.

Gopala, V. R. and van Wachem, B. G. M., 2008. Volume of fluid methods for immisciblefluid and free-surface flows. Chemical Engineering Journal, 141, 204-221.

Gu, Y. and Li, D., 1998. A model for a liquid drop spreading on a solid surface. Colloids and Surfaces A: Physicochemical and Engineering Aspects, 142, 243-256.

Gunjal, P. R., Ranadev, V. V., Chaudhari, R. V., 2003. Experimental and computational study of liquid drop over flat and spherical surfaces. Catalysis Today, 79-80, 267-273.

Hocking, L. M., 1992. Rival contact-angle models and the spreading of drops. Journal of Fluid Mechanics, 239, 671-681. 
Jasak, H. and Weller, H. G., 1995. Interface-tracking capabilities of the InterGamma differencing scheme. Technical Report, Imperial College, University of London.

Lunkad, S. F., Buwa, V.V., Nigam, K. D. P., 2007. Numerical simulations of drop impact and spreading on horizontal and inclined surfaces. Chemical Engineering Science, 62, 7214-7224.

Pont, V., Saleh, K., Steinmetz, D., Hemati, M., 2001. Influence of the physicochemical properties on the growth of solid particles by granulation in fluidized bed. Powder Technology, 120, 97-104.

Rafaï, S., Boudaoud, A., Bonn, D., 2004. Spreading of non-Newtonian fluids on hydrophilic surfaces. J. Fluid Mech., 513, 77-85.

Rajniak, P., Mancinelli, C., Chern, R., Stepanek, F., Farber, L., Hill, B., 2007. Experimental study of wet granulation in fluidized bed: Impact of the binder properties on the granule morphology. International Journal of Pharmaceutics, 334, 92-102.

Summ, B. D. and Samsonov, V. M., 1999. Concepts of Rehbinder's school and modern theories of spreading. Colloids and Surfaces A: Physicochemical and Engineering Aspects, 160, 63-77.

Tan, H. S., Salman, A. D., Hounslow, M. J., 2006. Kinetics of fluidised bed melt granulation I: The effect of process variables. Chemical Engineering Science, 61, 15851601.

Tanner, L. H., 1979. The spreading of silicone oil drops on horizontal surfaces. J. Phys. D: Applied Phys., 12, 1473-84.

Ubbink, O., 1997. Numerical Prediction of Two Fluid Systems With Sharp Interfaces. . Technology and Medicine. London, England, Imperial College of Science.

van Wachem, B. G. M., Schouten, J. C., 2004. Experimental validation of 3-D lagrangian VOF model: Bubble shape and rise velocity. AIChE, 48, 2744-2753. 


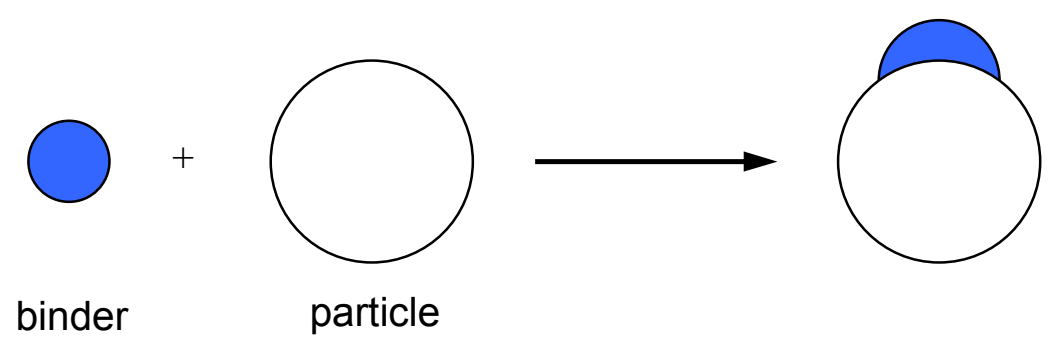

Fig. 1. Schematic of the droplet-particle collision process leading to the wetting of the particle.

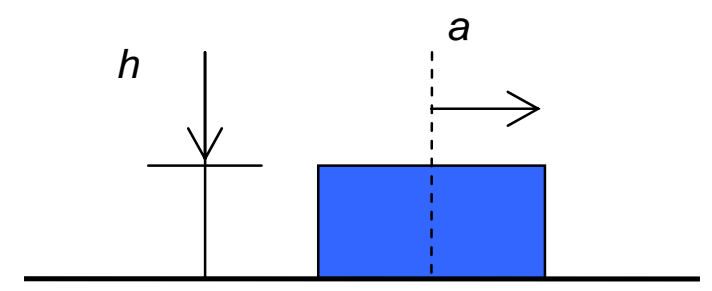

Fig. 2. Schematic of the approximate representation of a spreading drop. 


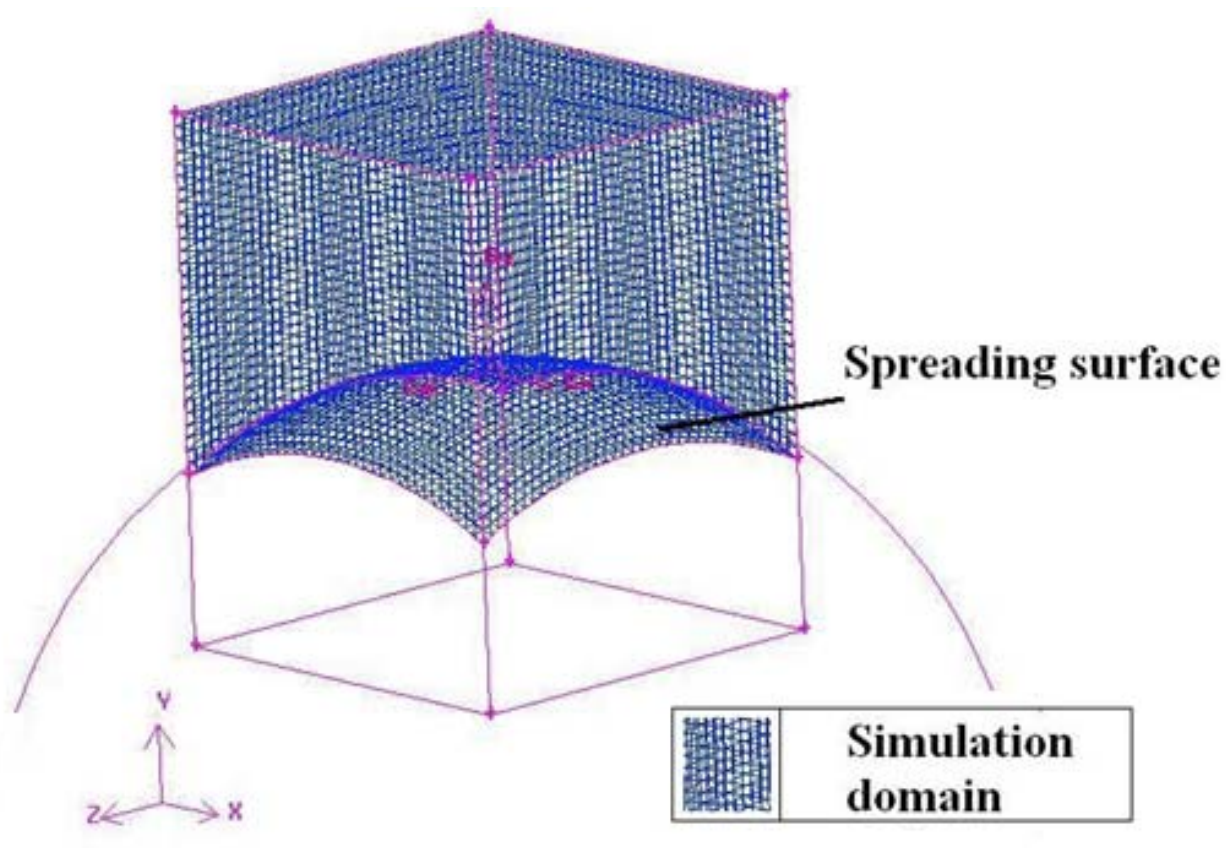

Fig. 3. shows a typical mesh volume of the computational domain used for the case of droplet on curved surface. 


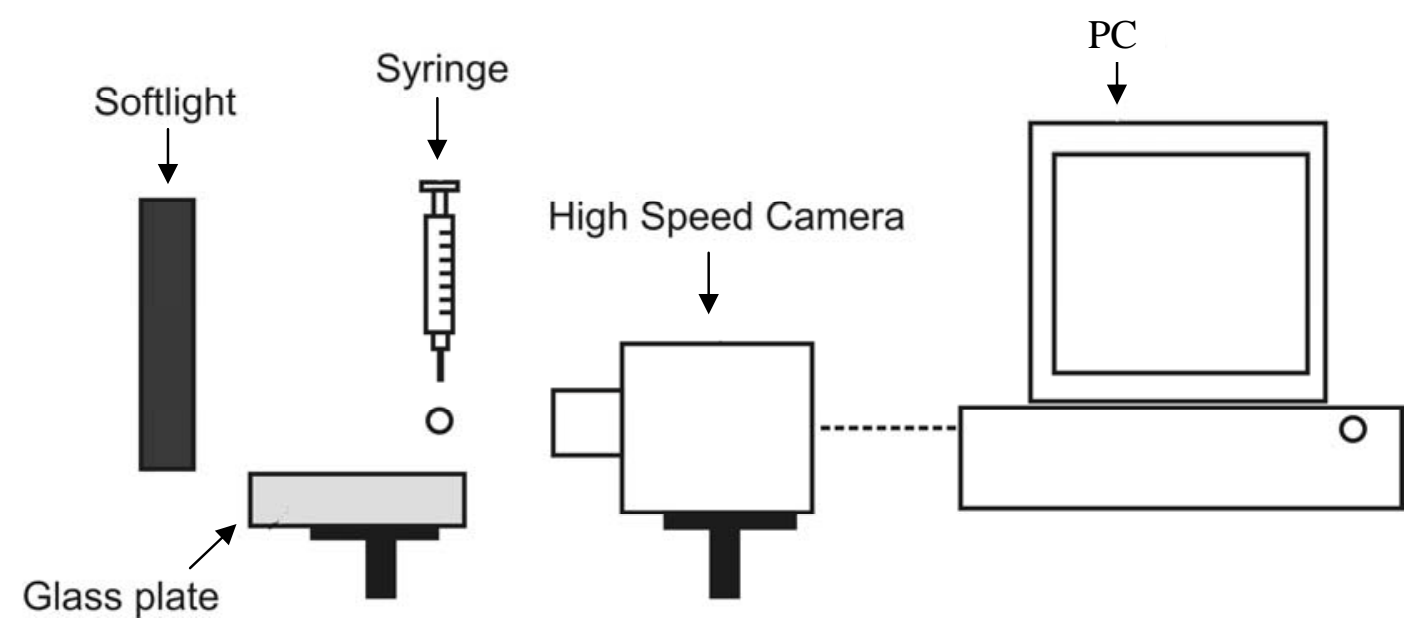

Fig. 4. Schematic representation of the experimental set-up 


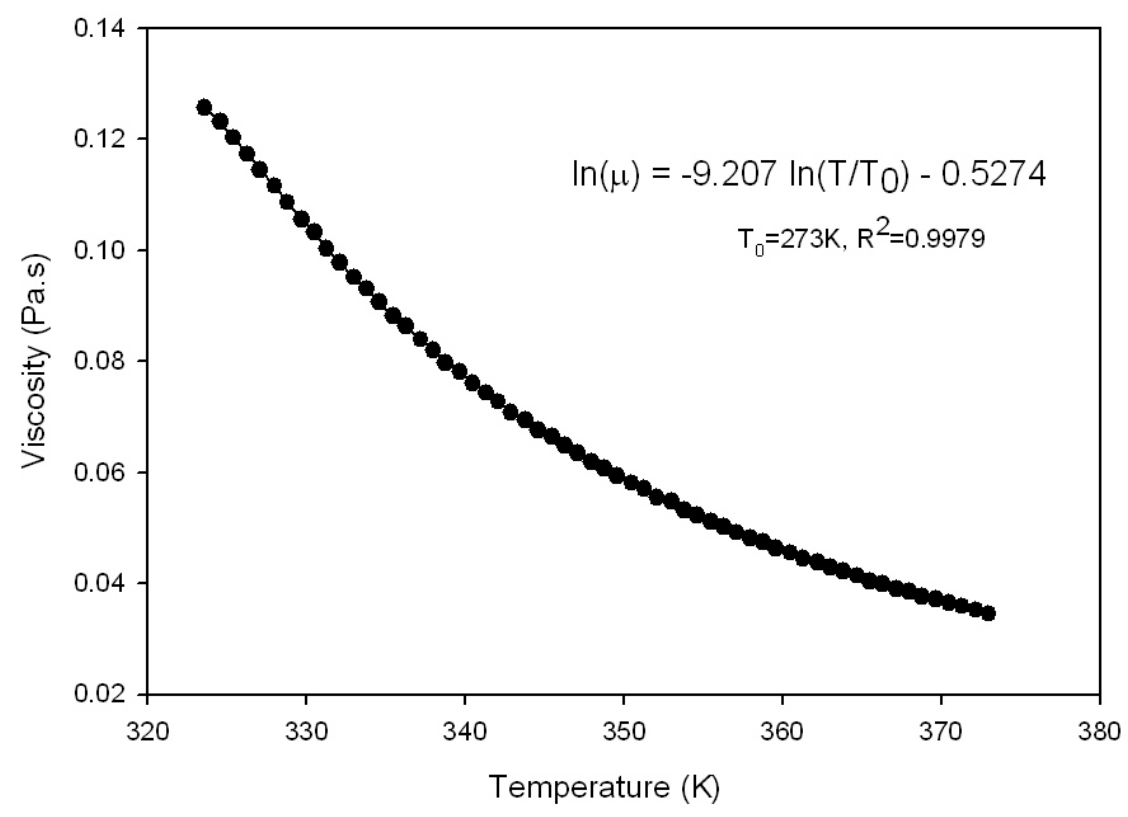

Fig. 5. Experimentally determined PEG1500 viscosity as function of temperature 
(a) Experiment

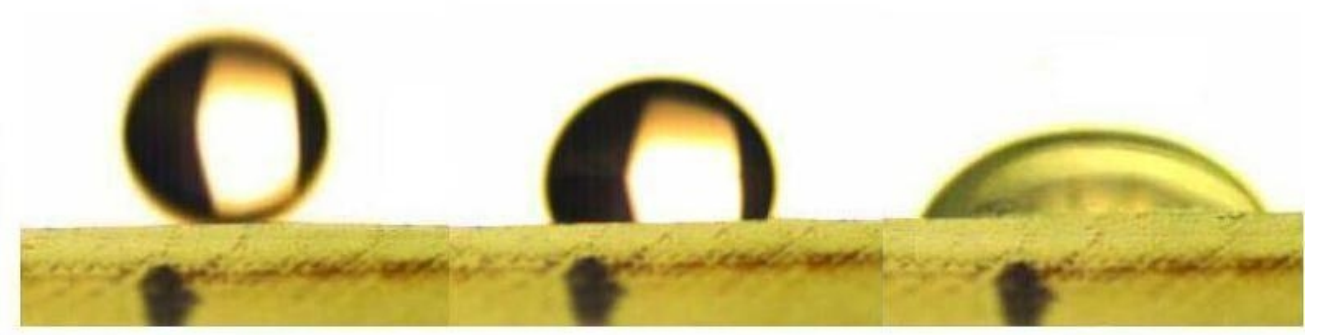

(b) Simulation

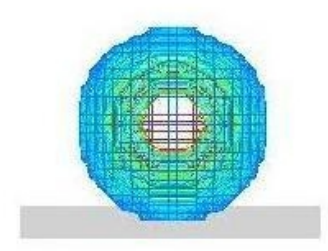

0.0s

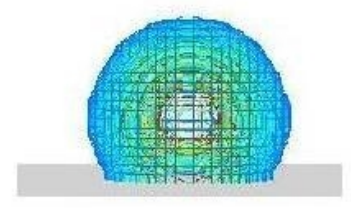

0.01s

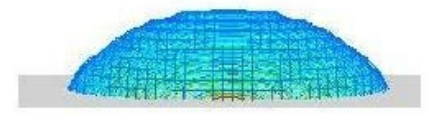

0.1s

Fig. 6. Comparison of experiment with VOF simulation for a $3 \mathrm{~mm}$ PEG1500 droplet spreading on a flat glass surface with an initial droplet temperature of $67^{\circ} \mathrm{C}$ 

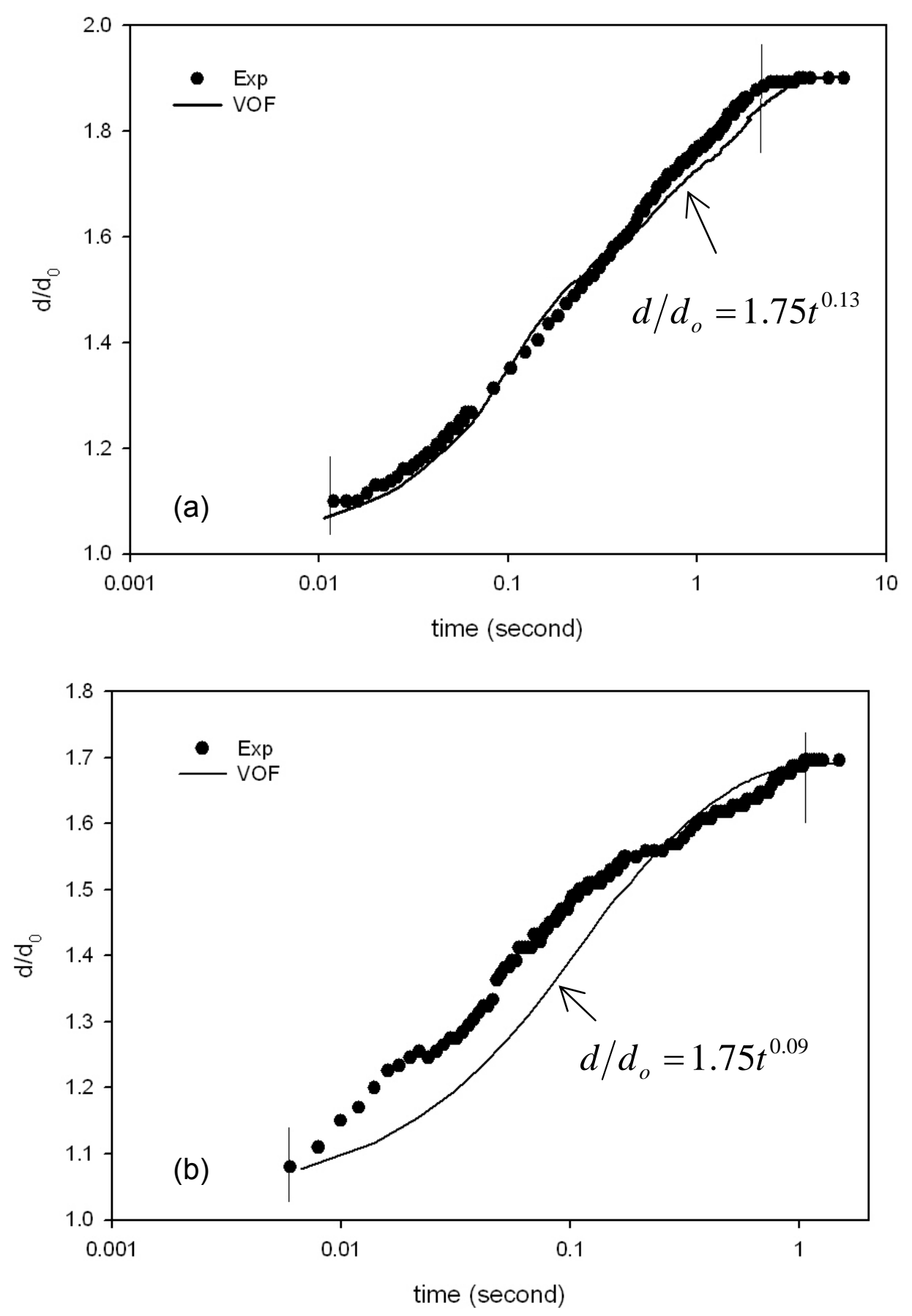

Fig. 7. Model prediction versus experiment result for a $3 \mathrm{~mm}$ PEG1500 droplet spreading over a glass surface. Initial droplet temperature (a) $50^{\circ} \mathrm{C}$ (b) $67^{\circ} \mathrm{C}$. 


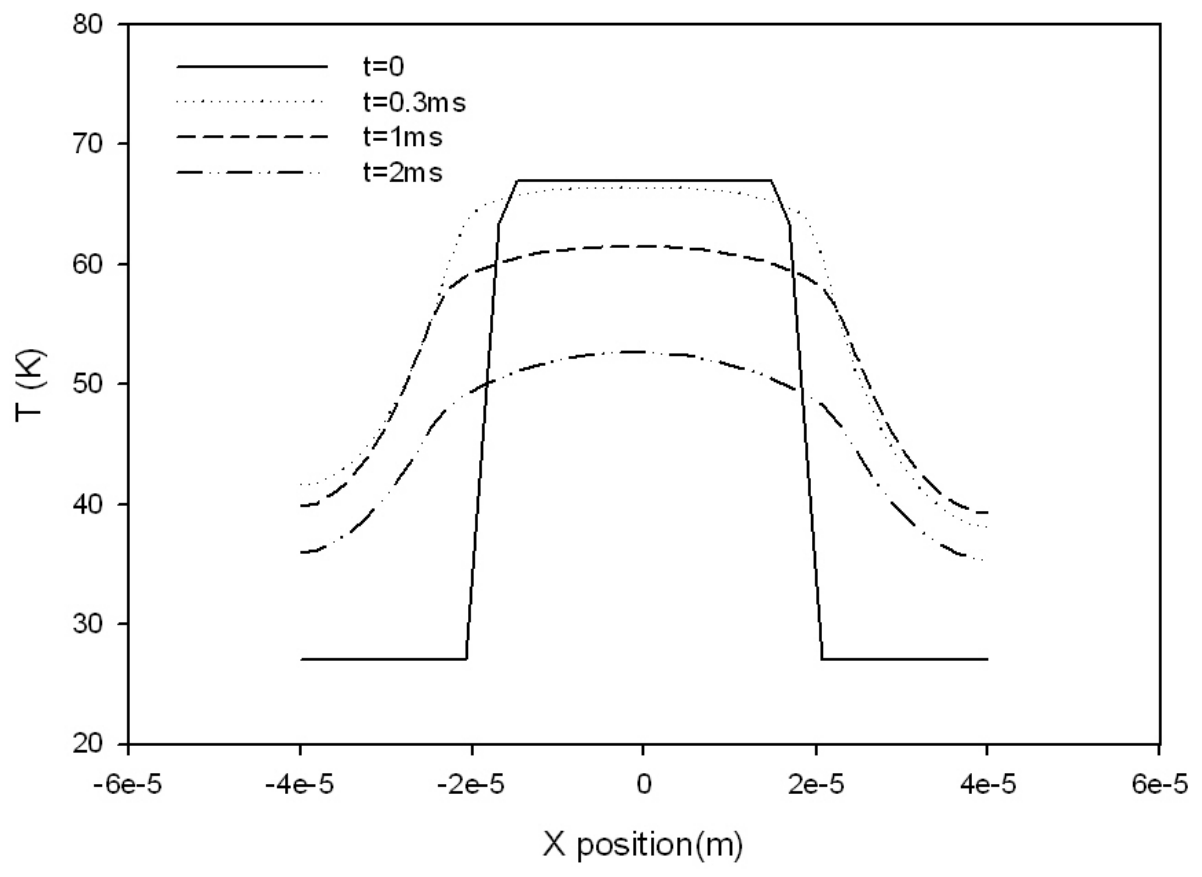

Fig. 8. Binder temperature profile at the binder spreading surface interface. Initial droplet condition of $40 \mu \mathrm{m}$ diameter and $67^{\circ} \mathrm{C}$ temperature. 


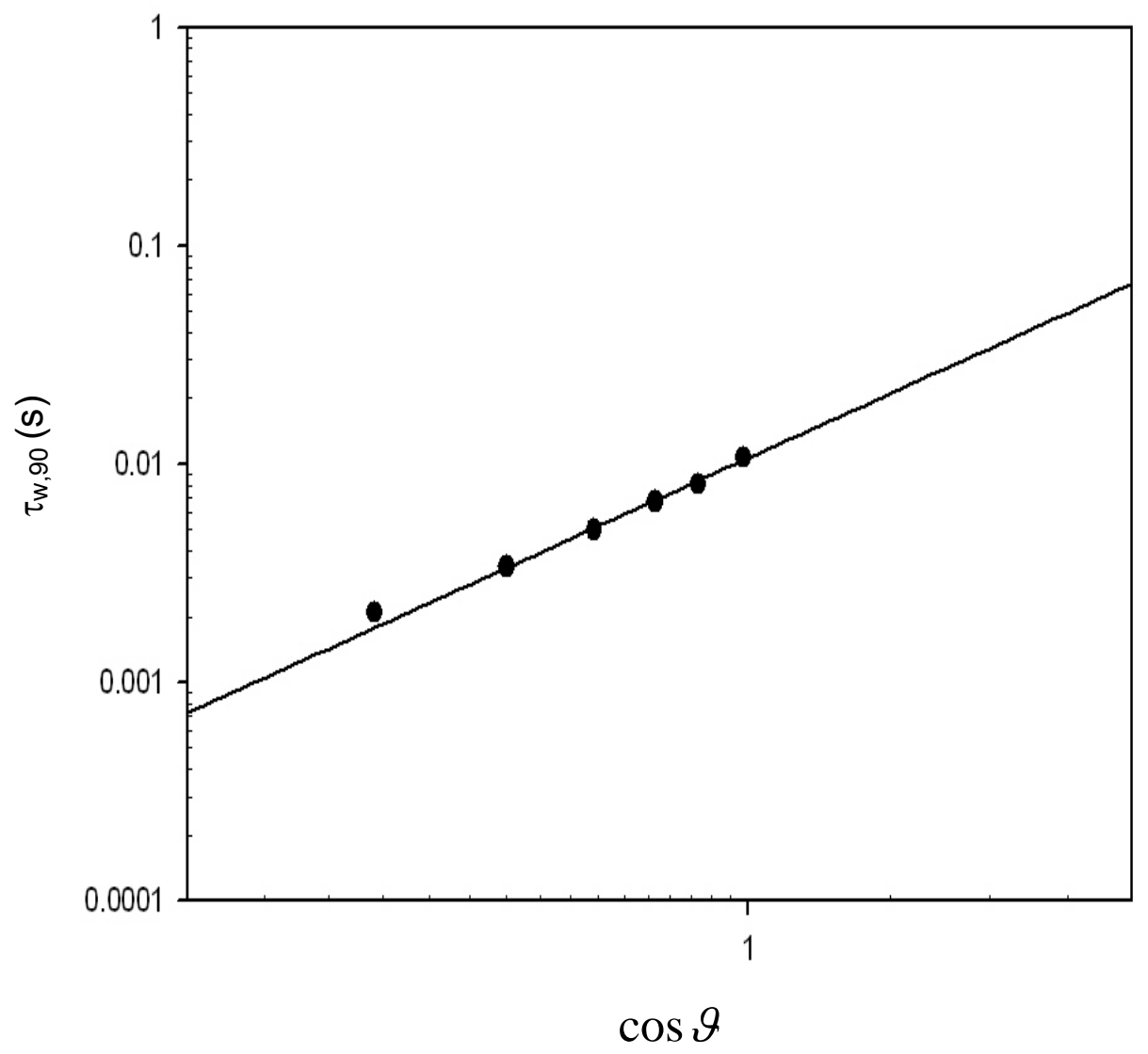

Fig. 9. Binder spreading rate as function of Contact angle for $0.1 \mathrm{~Pa}$.s viscosity, $0.01 \mathrm{~N} / \mathrm{m}$ surface tension, $3 \mathrm{D} / \mathrm{d}_{0}$. The points are VOF simulation results and the line is best fit. 


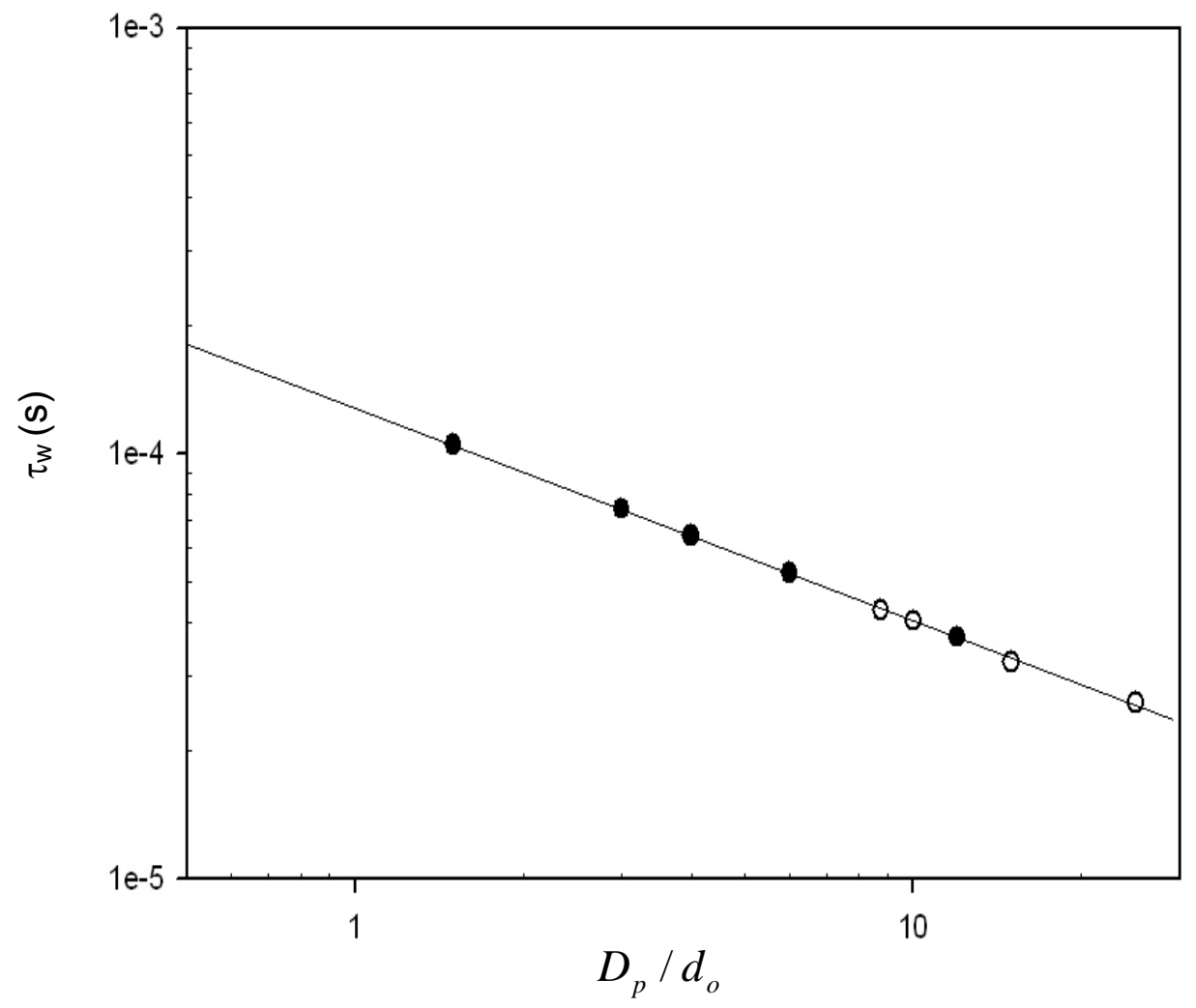

Fig.10. Spreading time as function of $D_{p} / d_{o}$ for 0.1 Pa.s viscosity, $0.15 \mathrm{~N} / \mathrm{m}$ surface tension, $60^{\circ}$ contact angle. Full circle, $\bullet$, refers to VOF simulation results for various droplet diameters, $d_{o}=10,20,30,40$, and $80, \mu \mathrm{m}$ at a fixed particle size, $D_{p}=120 \mu \mathrm{m}$. Open circle, o, refers to simulation results for various particle diameters, $D_{p}=175,200$, 300 and $500 \mu \mathrm{m}$, at a fixed droplet size, $d_{o}=20 \mu \mathrm{m}$. The line is best fit. 


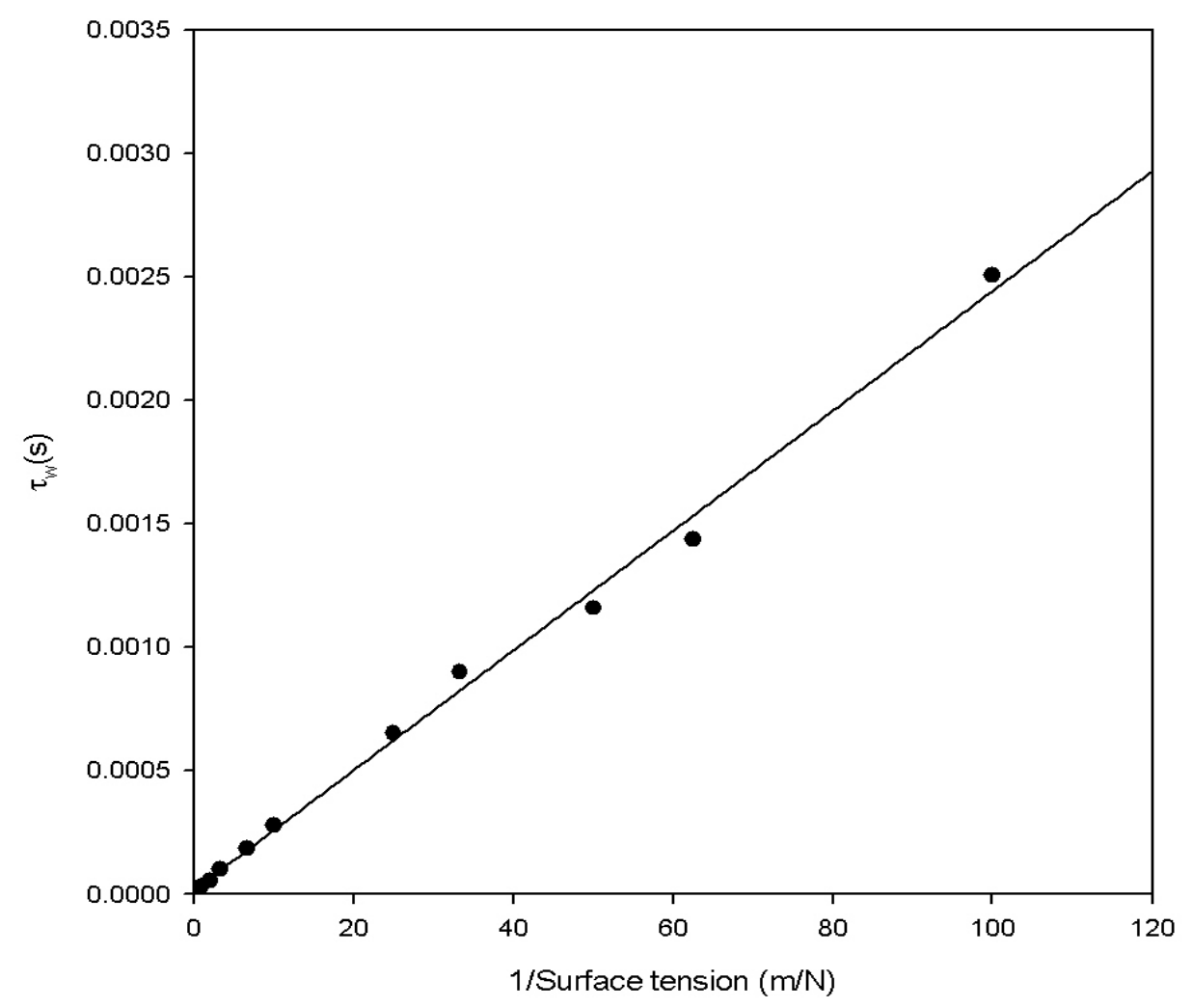

Fig. 11. Binder spreading rate as function of the inverse of the surface tension. $\mu=0.1$ Pa.s, $D_{p} / d_{o}=3$ and $\vartheta=60^{\circ}$. The points represent VOF simulation results and the line is best fit. 


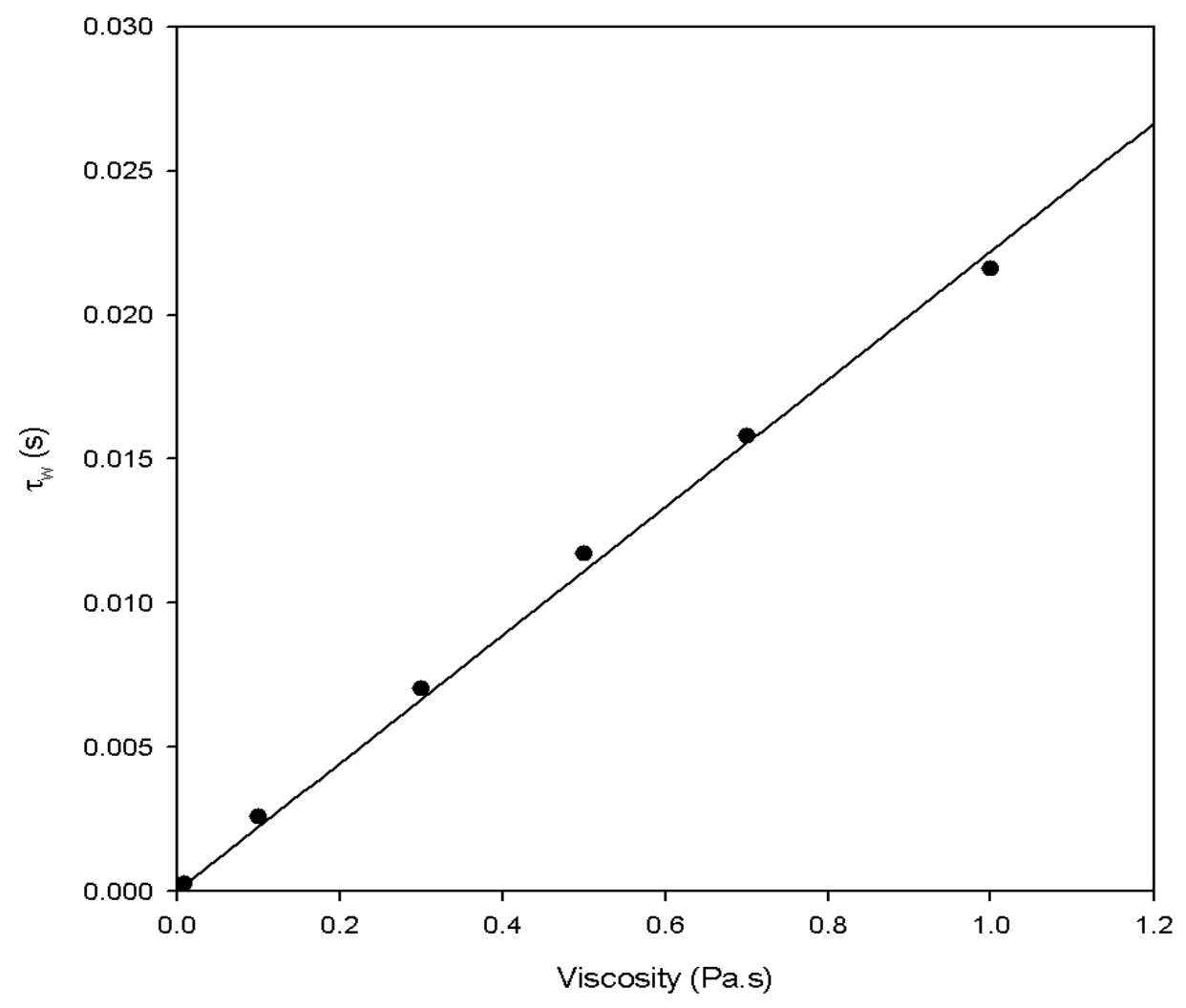

Fig. 12. Binder spreading rate as function of the binder viscosity. $\gamma=0.01 \mathrm{~N} / \mathrm{m}, D_{p} / d_{o}=$ 3 and $\vartheta=60^{\circ}$. The points represent VOF simulation results and the line is best fit. 


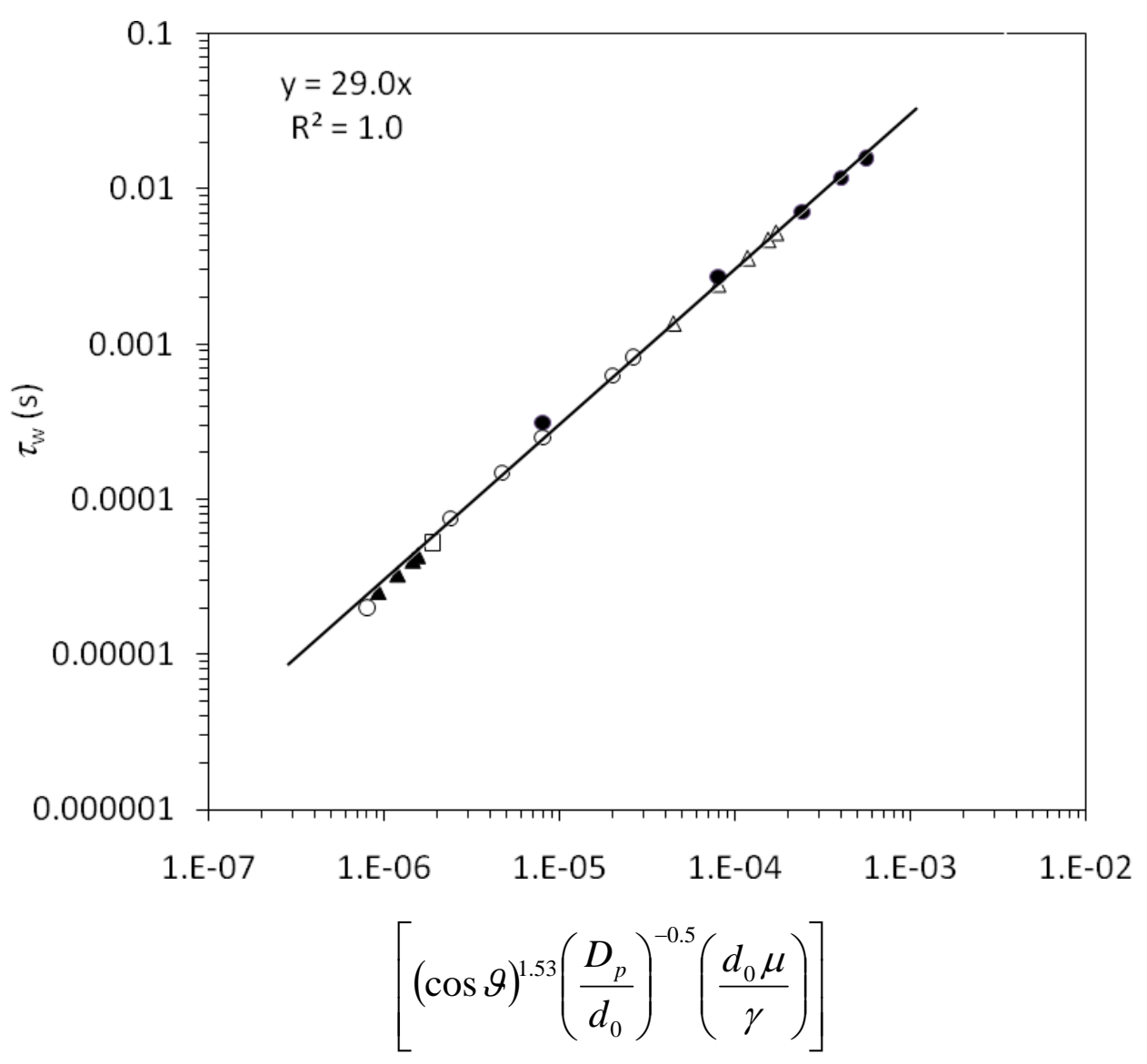

Fig. 13. Generalization of spreading time. The symbols refer to different VOF simulation conditions as shown in Table 2, the line is best fit. 

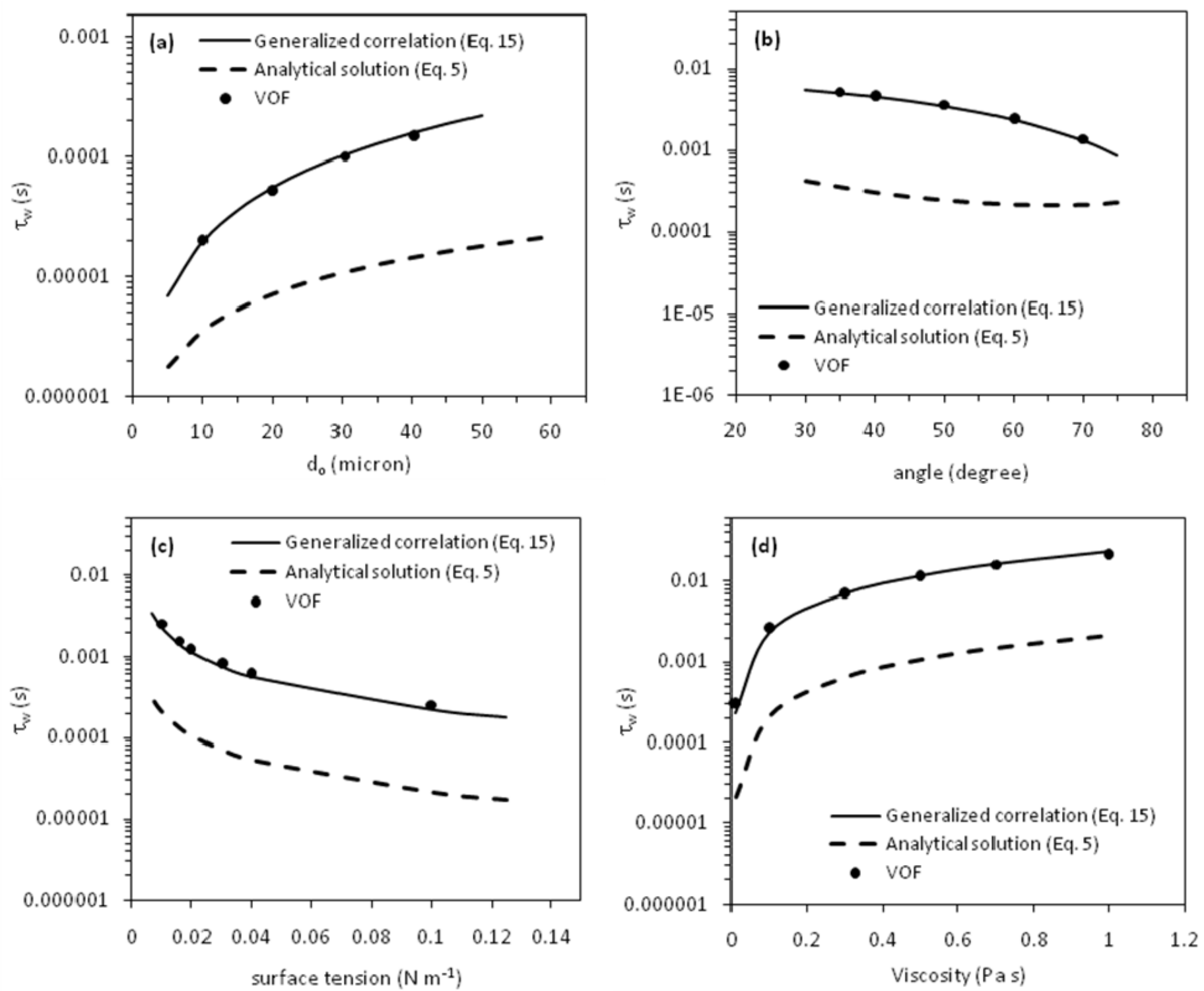

Fig. 14. A Comparison of binder spreading time obtained from the VOF, analytical solution and the generalized correlation (a) $d_{o}=20 \mu \mathrm{m}, \mu=0.1$ Pa.s, $\gamma=0.15 \mathrm{~N} . \mathrm{m}^{-1}$,

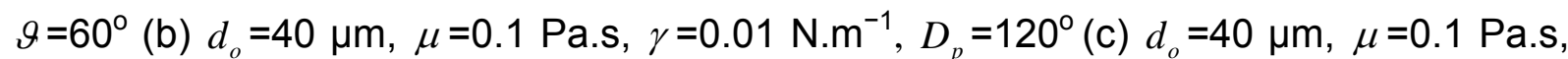
$\vartheta=60^{\circ}, D_{p}=120^{\circ}$ (d) $d_{o}=40 \mu \mathrm{m}, \gamma=0.01 \mathrm{~N} \cdot \mathrm{m}^{-1}, \vartheta=60^{\circ}, D_{p}=120^{\circ}$ 
Table 1. PEG1500 binder properties

\begin{tabular}{l|l}
\hline Material & PEG1500 \\
\hline Viscosity (Pa.s) & $0.109^{a}$ \\
Surface tension $\left({\left.\mathrm{N} . \mathrm{m}^{-1}\right)}\right)$ & $0.15^{b}$ \\
Density $\left(\mathrm{kg} / \mathrm{m}^{3}\right)$ & $1093^{b}$ \\
Melting temperature $\left({ }^{\circ} \mathrm{C}\right)$ & $\sim 45.0$ \\
\hline${ }^{a}$ Measured at $55^{\circ} \mathrm{C}$ (This study) \\
${ }^{b}$ Measured at $60^{\circ} \mathrm{C}$ (Tan et al., 2006)
\end{tabular}

Table 2. Simulation parameters used to estimate spreading time shown in Fig 13.

\begin{tabular}{|l|c|c|c|c|c|}
\hline & $\bullet$ & $\circ$ & $\Delta$ & $\Delta$ & $\square$ \\
\hline$\mu($ Pa.s $)$ & $0.01-0.7$ & 0.1 & 0.1 & 0.1 & 0.1 \\
\hline$\gamma\left(\mathrm{N} . m^{-1}\right)$ & 0.01 & $0.02-1.0$ & 0.15 & 0.15 & 0.1 \\
\hline$D_{p}(\mu \mathrm{m})$ & 120 & 120 & $175-500$ & 120 & 120 \\
\hline$d_{0}(\mu \mathrm{m})$ & 40 & 40 & 40 & 20 & 40 \\
\hline$\vartheta($ degree $)$ & 60 & 60 & 60 & 60 & $35-70$ \\
\hline
\end{tabular}

Table 3. Properties of commonly used binders and the corresponding estimated spreading time using the generalized correlation Eq. 15

\begin{tabular}{l|c|c|c|c}
\hline \multicolumn{1}{c|}{ Binder } & $\begin{array}{c}\text { angle } \\
(\text { degree })\end{array}$ & $\begin{array}{c}\text { Surface tension } \\
\left(\text { N.m }^{-1}\right)\end{array}$ & $\begin{array}{c}\text { Viscosity } \\
(\text { Pa.s })\end{array}$ & $\begin{array}{c}\text { Spreading time } \\
(\mathrm{s})\end{array}$ \\
\hline PEG1500 & $35^{a}$ & $0.15^{b}$ & $0.109^{a}$ & $2.86 \times 10^{-4}$ \\
CMC 1\% ${ }^{c}$ & 72 & 0.72 & 0.112 & $1.38 \times 10^{-5}$ \\
HPC 5\% ${ }^{d}$ & 26 & 0.43 & 0.0195 & $2.06 \times 10^{-5}$ \\
HPC 10\% ${ }^{d}$ & 46 & 0.43 & 0.164 & $1.17 \times 10^{-4}$ \\
\hline
\end{tabular}

\footnotetext{
${ }^{a}$ Measured at $55^{\circ} \mathrm{C}$ (This study)

${ }^{b}$ Measured at $60^{\circ} \mathrm{C}$ (Tan et al., 2006)

${ }^{c}$ Obtained from Pont et al. (2001)

${ }^{d}$ Obtained from Rajnaik et al. (2007)
} 